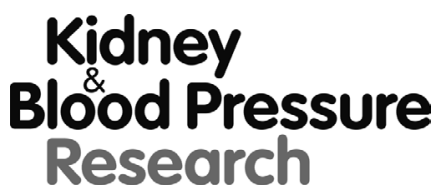

Kidney Blood Press Res 2018;43:979-986

DOI: $10.1159 / 000490687$

Published online: 19 June, 2018

Accepted: 7 June, 2018

This article is licensed under the Creative Commons Attribution-NonCommercial-NoDerivatives 4.0 InternaThis article is licensed under the Creative Commons Attribution-NonCommercial-NoDerivatives 4.0 Interna-
tional License (CC BY-NC-ND) (http://www.karger.com/Services/OpenAccessLicense). Usage and distribution tional License (CC BY-NC-ND) (http://www.karger.com/Services/OpenAccessLicense). Usage and dist

\title{
Effect of Home Blood Pressure Monitoring on Patient's Awareness and Goal Attain- ment Under Antihypertensive Therapy: The Factors Influencing Results in Anti- HypertenSive Treatment (FIRST) Study
}

\author{
David Spirk ${ }^{a}$ Sarah Noll ${ }^{b}$ Michel Burnier ${ }^{c}$ Stefano Rimoldid Georg Noll ${ }^{b, e}$ \\ Isabella Sudanob,f \\ anstitute of Pharmacology, University of Bern, Bern, bUniversity of Zurich, Zurich, 'Service of Nephrol- \\ ogy and Hypertension, Lausanne University Hospital, Lausanne, dDepartment of Cardiology, Bern Uni- \\ versity Hospital, University of Bern, Bern, eHeart Clinic Hirslanden, Zurich, fDepartment of Cardiology, \\ University Heart Center, University Hospital Zurich, Zurich, Switzerland
}

\section{Key Words}

Arterial hypertension • Antihypertensive therapy • Goal attainment $\bullet$ Home blood pressure monitoring • Tolerability

\begin{abstract}
Background/Aims: Despite availability of a broad spectrum of blood pressure (BP)-lowering drugs many hypertensive patients do not attain BP goals. We aimed to evaluate the influence of home blood pressure monitoring (HBPM) on patient's awareness and attainment of BP goals under antihypertensive treatment with irbesartan alone or in combination with hydrochlorothiazide. Methods: In total, 1,268 patients with arterial hypertension were enrolled in the Factors Influencing Results in anti-hypertenSive Treatment (FIRST) study by 348 general practitioners and internal medicine specialists across Switzerland. Patients selected for HBPM received detailed information and training on BP self-management. The study endpoints included patient's awareness and attainment of BP goals, and the efficacy and tolerability of antihypertensive treatment at 3 months. Results: Overall, the mean age was $61 \pm 13$ years and 616 (49\%) were women. The mean systolic/diastolic BP was $161 \pm 17 / 96 \pm 11 \mathrm{mmHg}$, and 239 (19\%) patients had diabetes mellitus. $758(60 \%)$ patients were instructed to use HBPM. Both the proportion of patients aware of their BP goals (81\% vs. $70 \% ; p<0.001)$ and the percentage of patients reaching their BP goal (64\% vs. $57 \% ; p=0.028)$ were higher in those with vs. without HBPM. The mean reduction in systolic/diastolic BP was $23.8 / 13.2 \mathrm{mmHg}$. Only 35
\end{abstract}

D. Spirk und S. Noll equally contributed to this work.

Isabella Sudano, MD PhD

Department of Cardiology, University Heart Center, University Hospital Zurich

Rämistrasse 100, $\mathrm{CH}-8091$ Zurich (Switzerland)

Tel. +41 4425558 41, Fax +41 4425548 59, E-Mail isabella.sudano@usz.ch 


\section{Kidney Blood Pressure Research}

(3.0\%) patients discontinued antihypertensive therapy. Conclusion: In a large Swiss cohort of patients with arterial hypertension, information and training on BP self-measurement and direct involvement of patients by using HBPM led to improvement in BP control. Treatment with irbesartan alone or in combination with hydrochlorothiazide was well tolerated and markedly reduced $B P$.

\section{Introduction}

Hypertension is one of the main risk factors for cardiovascular disease. Nevertheless, despite treatment a substantial number of patients do not attain blood pressure (BP) goals.

Hypertensive patients are usually treated by family physicians and clinical trials, often performed in hospital setting, do not necessarily reflect daily clinical practice. In clinical practice, many reasons may explain the non-attainment of BP target value; among these, insufficient dosage of antihypertensive drugs, combination of drugs which do not have additional effect together with poor tolerability, and insufficient compliance should be considered [1]. Frequent changes of antihypertensive therapy may not be a good strategy as suggested by the results of the "Reason for Not Intensifying Antihypertensive Treatment" (RIAT) study [2].

Particularly, patient non-adherence is known to be an important factor [3-5]. Previous studies have shown that home blood pressure monitoring (HBPM) is effective for improving compliance in the treatment of hypertension [6]. Even though the time relative to a visit to the doctor seems to be an important predictor of patients' adherence to treatment [7], the HBPM could work as a supporting factor to improve the patients' compliance. A proper education and empowerment of patients is essential for successful treatment of chronic diseases, such as hypertension: well informed patients which regularly use HBPM are more compliant and therefore reach their individual BP goals more easily. In addition, HBPM is known to be useful for the initial diagnosis and long-term follow-up of treated hypertension, especially in regard to the white-coat effect [8].

The present study aims primarily to evaluate whether HBPM influences patient's awareness and attainment of BP goals under standard or intensified antihypertensive treatment with irbesartan monotherapy or combination therapy (irbesartan plus hydrochlorothiazide).

\section{Materials and Methods}

General practitioners (GPs) and internal medicine specialists across all regions of Switzerland were invited to screen and enroll up to 5 patients per physician during a 10-months period between July 2005 and April 2006. For the purpose of representativeness, Switzerland was divided into 10 geographic sectors of similar territorial and population size, and for each of these sectors, 30-36 physicians were randomly selected from a centralized national register and asked to participate in this prospective cohort study. Inclusion criteria were age of $\geq 18$ years and newly diagnosed or previously treated uncontrolled arterial hypertension. No exclusion criteria were defined. Written informed consent was obtained.

At the baseline visit, patient characteristics (such as age, sex, weight, height, and current systolic and diastolic arterial BP), cardiovascular risk factors, and clinically confirmed cardiovascular disease were recorded.

Cardiovascular risk factors were defined as smoking, dyslipidemia (total cholesterol $>6.5 \mathrm{mmol} / \mathrm{l}$ ), diabetes mellitus, a positive family history of cardiovascular disease, an elevated C-reactive peptide ( $>1 \mathrm{mg} /$ $\mathrm{dl}$ ), and abdominal obesity (waist circumference $>102 \mathrm{~cm}$ in men and $>88 \mathrm{~cm}$ in women). End organ damage was defined as left ventricular hypertrophy detected by electrocardiography (Sokolow-Lyons $>38 \mathrm{~mm}$, Cornell $>2440 \mathrm{~mm} * \mathrm{~ms}$ ) or echocardiography (left ventricular mass index $\geq 125 \mathrm{~g} / \mathrm{m}^{2}$ in men and $\geq 110 \mathrm{~g} / \mathrm{m}^{2}$ in women), atherosclerosis (ultrasound evidence of arterial wall thickening [carotid intima-media thickness $\geq 0.9 \mathrm{~mm}$ ] or atherosclerotic plaque), and/or renal damage (microalbuminuria of $30-300 \mathrm{mg} / 24 \mathrm{~h}$, or serum creatinine of $115-133 \mu \mathrm{mol} / \mathrm{l}$ in men and 107-124 $\mu \mathrm{mol} / \mathrm{l}$ in women). 


\section{Kidney Blood Pressure Research}

Clinically confirmed cardiovascular disease was defined as cerebrovascular disease (stroke, transient ischemic attack), heart disease (myocardial infarction, angina pectoris, coronary revascularization, heart failure), kidney disease (diabetic and non-diabetic nephropathy), renal failure (serum creatinine $>133$ $\mu \mathrm{mol} / \mathrm{l}$ in men and $>124 \mu \mathrm{mol} / \mathrm{l}$ in women), vascular disease (aneurysm, peripheral artery disease), and/or advanced hypertensive retinopathy (hemorrhage, exudates, or papillary edema).

According to the then contemporary guidelines [9], office blood pressure measurements were used to categorize BP as normal (systolic 120-129 mmHg or diastolic 80-84 mmHg), high normal (systolic 130-139 mmHg or diastolic 85-89 mmHg), mild hypertension (systolic 140-159 mmHg or diastolic 90-99 mmHg), moderate hypertension (systolic 160-179 mmHg or diastolic 100-109 $\mathrm{mmHg}$ ), and severe hypertension (systolic $>180 \mathrm{mmHg}$ or diastolic $>110 \mathrm{mmHg}$ ).

At the baseline visit, an individual BP goal according to the current BP and the individual risk profile was defined, and a suitable antihypertensive medication and the use of HBPM were then chosen at discretion of treating physicians. Specifically, the individual BP goal was set to lower both systolic and diastolic BP intensively, at least below 140/90 $\mathrm{mmHg}$ in all hypertensive patients, and below 130/80 $\mathrm{mmHg}$ in diabetics and patients with chronic kidney disease and proteinuria. The prescribed medication included monotherapy with irbesartan (150 or $300 \mathrm{mg}$ q.d.), combination therapy with irbesartan plus hydrochlorothiazide (HCTZ) (150 or 300 mg irbesartan +12.5 mg HCTZ q.d.), or other antihypertensives. Patients with HBPM received detailed information and training on BP self-management and its documentation. Briefly, the patients were instructed to measure their BP once a week and on 6 consecutive days prior to each physician's visit, in the morning, before drug intake, in a quiet room, and seated. The measurements were supposed to be done after rest for at least 5 minutes and after more than 30 minutes without smoking, meal or caffeine intake, or physical exercise. For the HBPM, Microlife device BP 3AC1-1PC, Average Mode (automatic data analysis of three successive measurements) was used. The patients were trained by their physicians how to use the device and could test it during the baseline visit. During the study period, the results of the HBPM were saved directly on the device for the physicians to assess and control [10].

The primary endpoint was patient's awareness of BP goals and BP goal attainment at the follow-up visit after 3 months. Secondary endpoints included control of systolic and diastolic BP, and satisfaction with the HBPM device.

Continuous variables with a normal distribution are presented as means with standard deviations (SD), and group comparisons were performed with the t-test; continuous variables with skewed distribution are presented as median values with interquartile ranges (IQR), and group comparisons were performed using a rank-sum test. Discrete variables are presented as frequencies and percentages, and group comparisons were performed with the chi square or Fisher's exact test. All p-values are two-tailed. Data were analyzed using the STATA 9 software (STATACorp LP, College Station, Texas, USA). Ethics committee approvals were obtained according to local regulations valid at the time of study conduct.

\section{Results}

Overall, 348 physicians participated in the study and enrolled 1, 268 patients with arterial hypertension. The clinical characteristics of the studied population are summarized in Table 1 ; the mean age was $61 \pm 13$ years, $616(49 \%)$ were women, and the mean BMI was $28 \pm 5 \mathrm{~kg} / \mathrm{m}^{2}$. The mean systolic BP was $161 \pm 17 \mathrm{mmHg}$, the mean diastolic BP was $96 \pm 11$ $\mathrm{mmHg}$, and 239 (19\%) patients had diabetes mellitus.

The majority of patients started a new BP-lowering medication within a week after the baseline visit, while 100 (8\%) patients continued their established antihypertensive therapy. 32\% of the patients received irbesartan $150 \mathrm{mg}$ monotherapy, $12 \%$ irbesartan $300 \mathrm{mg}$ monotherapy, 25\% irbesartan $150 \mathrm{mg}$ plus $12.5 \mathrm{mg}$ HCTZ combination therapy, 16\% irbesartan $300 \mathrm{mg}$ plus $12.5 \mathrm{mg}$ HCTZ combination therapy, and 15\% other BP-lowering therapy.

At the baseline visit, $758(60 \%)$ patients were instructed to use HBPM to control their blood pressure regularly at home. HBPM was more frequently used by younger patients aged $\leq 55$ years compared to older patients aged $>71$ years $(66 \%$ vs. $54 \%, p=0.041)$. The mean BMI was similar between patients with vs. without HBPM $\left(28.3 \pm 4.8\right.$ vs. $28.5 \pm 4.9 \mathrm{~kg} / \mathrm{m}^{2}$; $\mathrm{p}=0.47$ ). Among patients with HBPM, there was no significant difference in gender (female $59 \%$ vs. male 63\%; p=0.10), body weight ( $\leq 70 \mathrm{~kg} \mathrm{60 \% ,71-80} \mathrm{kg} \mathrm{57 \% ,} \mathrm{81-90} \mathrm{kg} \mathrm{66 \% ,} \mathrm{>90}$

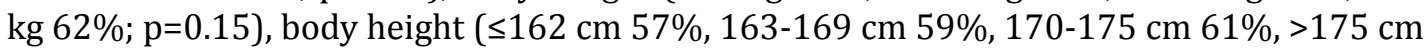




\section{Kidney \\ Blood Pressure Research}

Kidney Blood Press Res 2018;43:979-986

\begin{tabular}{l|l}
\hline DOI: $10.1159 / 000490687$ & (c) 2018 The Author(s). Published by S. Karger AG, Basel
\end{tabular}

Published onlıne: 19 June, 2018

www.karger.com/kb
$67 \% ; \mathrm{p}=0.10$ ), and presence of additional cardiovascular risk factors (none $63 \%$, one or two $62 \%$, $\geq$ three $58 \% ; \mathrm{p}=0.52$ ).

In total, 1,151 patients showed up for the follow-up appointment at 3 months. The mean \pm SD duration from the baseline to the follow-up visit was $99 \pm 39$ days, and the median (IQR) duration was 94 (86-107) days.

At the follow-up visit after 3-months, $61 \%$ of all patients managed to reach their BP goals (systolic and diastolic). The percentage of patients reaching their BP goal was higher in those with vs. without HBPM ( $64 \%$ vs. $57 \%$; $p=0.028$ ). At 3 months, $76 \%$ of all patients were aware of their BP goal set at the baseline visit. The patient's awareness of their BP goals was higher in those with vs. without HBPM (81\% vs. 70\%; $\mathrm{p}<0.001)$.

In total, the mean reduction in systolic BP was $23.8 \mathrm{mmHg}$ and the mean reduction in diastolic BP was $13.2 \mathrm{mmHg}$. The combination therapy of irbesartan plus HCTZ had the strongest effect on both systolic and diastolic BP, followed by the monotherapy with irbesartan and other antihypertensive therapy (Fig. 1, Fig. 2).

Between women and men, the mean reduction was similar in both systolic $(24.0 \mathrm{mmHg}$ vs. $22.3 \mathrm{mmHg}$; $\mathrm{p}=0.08)$ and diastolic $(11.9 \mathrm{mmHg}$ vs. $12.8 \mathrm{mmHg}$; $=0.18) \mathrm{BP}$, respectively. Regarding age, effects on drop in systolic BP were similar, although there was a difference regarding lowering of diastolic BP; patients under the age of 40 had a mean reduction of diastolic BP of $14.7 \mathrm{mmHg}$ compared to $13.2 \mathrm{mmHg}$ in patients of age between 40-60 and 11.5 $\mathrm{mmHg}$ in patients over the age of $60(\mathrm{p}=0.01)$. Similar results were observed when the blood pressure reduction was evaluated in patients with and without abdominal obesity; no difference in systolic BP reduction was observed, while the reduction in diastolic BP was significantly stronger in patients without vs. with abdominal obesity ( $13.1 \mathrm{vs.} 11.8 \mathrm{mmHg}$; $=0.04$ ).

The mean systolic BP was lowered to $138 \pm 13 \mathrm{mmHg}$ in patients with HBPM and $139 \pm 14$ $\mathrm{mmHg}$ in patients without HBPM $(\mathrm{p}=0.046)$. The mean diastolic BP was lowered to $83 \pm 9$ $\mathrm{mmHg}$ in patients with HBPM and $84 \pm 9 \mathrm{mmHg}$ in patients without HBPM $(\mathrm{p}=0.41)$.

Among patients with HBPM, 89\% were satisfied or very satisfied with the device used for the BP measurements. Satisfaction with the device was similar between genders (male $90 \%$ vs. female $89 \%$; $\mathrm{p}=0.75$ ) and across age categories ( $<40$ years $91 \%, 40-60$ years $91 \%$, $>60$ years $88 \%$; $\mathrm{p}=0.34$ ).

Overall, $35(3.0 \%)$ patients discontinued antihypertensive therapy during the course of the study. Of those, 9 patients stopped the treatment due to its insufficient effect, 5 due to side effects, 5 due to insufficient compliance, and 16 for other reasons. The rate of therapy discontinuation was similar in the HBPM-group compared to the non-HBPM-group $(3.6 \%$ vs. $2.1 \%$; $\mathrm{p}=0.20$ ).

\section{Discussion}

HBPM is being increasingly used in many health care systems world-wide, it is cost-effective and well accepted by patients [11-12]. This kind of monitoring is useful in many situations including appropriate adjustment of drug therapy in patients who have been previously misdiagnosed or over-treated. It promotes medication adherence and compliance and lifestyle changes, and helps to make patients more aware of their condition, determine 


\section{Kidney Blood Pressure Research}

Fig. 1. Reduction in the systolic blood pressure between treatment groups. $\mathrm{p}<0.05$ for interaction between treatment groups.

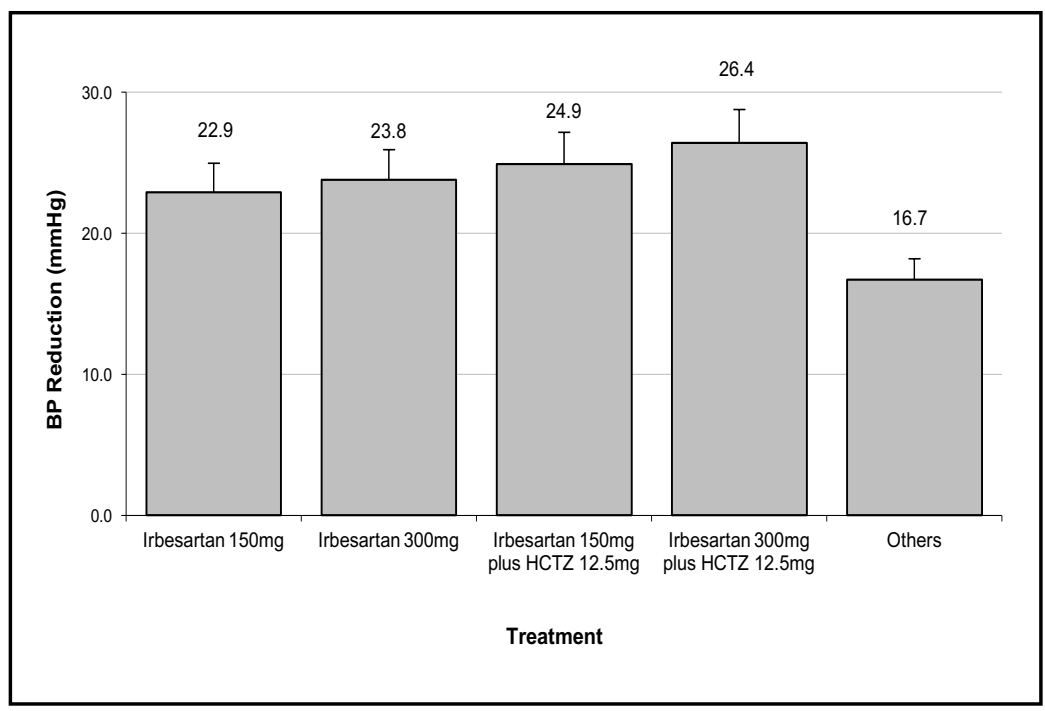

Fig. 2. Reduction in the diastolic blood pressure between treatment groups. $\mathrm{p}<0.05$ for interaction between treatment groups.

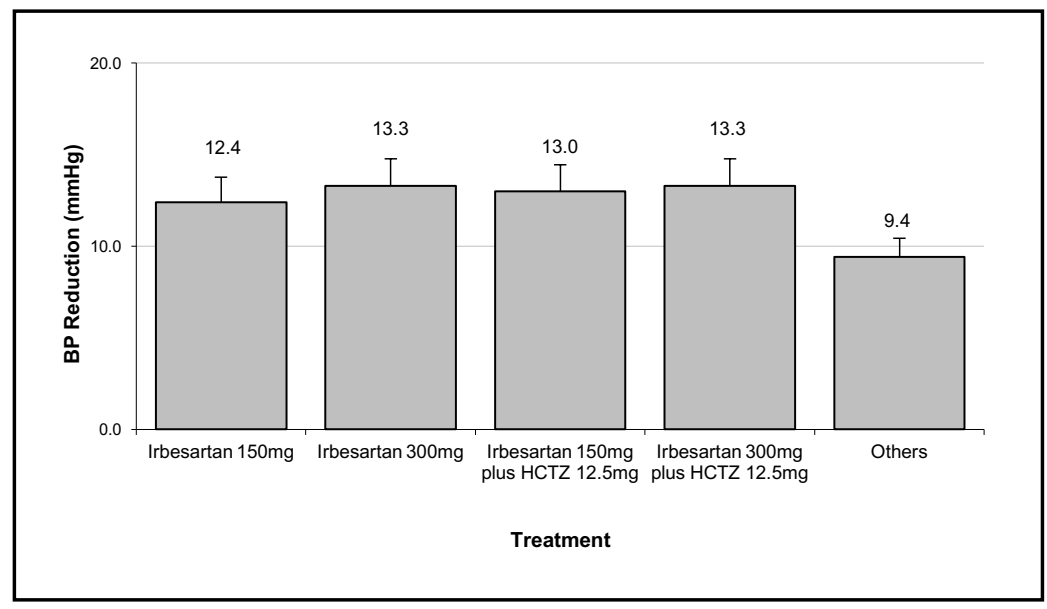

the efficacy of antihypertensive therapy, and support appropriate adjustment, particularly in hypertensive patients with white coat effect or in those with therapy-resistant hypertension [12-13]. To further improve the validity of HBPM, all patients in our study received detailed information and training on BP self-management with automatic documentation of all blood pressure values on the device used for HBPM.

The results of this observational study including a large population of hypertensive patients living in Switzerland show that promoting HBPM lead to a better disease awareness and persistence to therapy, and as a consequence, to a better BP control. In fact, even if antihypertensive drugs were well balanced in both groups, a better blood pressure control was observed in the group instructed for the use of HBPM as compared to the group without instructions for the HBPM use.

Improved patient awareness of the importance of an increased blood pressure has been identified as one of the major public health and medical challenges in the prevention and treatment of hypertension because of its potential beneficial impact on morbidity and mortality associated with cardiovascular disease and stroke [14]. Moreover, awareness of arterial hypertension as a cardiovascular risk factor was also associated with a better control of blood pressure in the group of patients with multiple cardiovascular risk factors in our study.

Even if HBPM is not suitable for all hypertensives, HBPM should not be considered contraindicated in patients with motor disabilities or cognitive impairments [12]. These 


\section{Kidney \\ Blood Pressure Research}

patients may be able to execute HBPM with additional support, either from a trained healthcare professional or family member. Moreover, HBPM performed with most automated devices is contraindicated in patients with pulse irregularities, such as atrial fibrillation [12]. The use of other monitoring tools, such as ambulatory blood pressure monitoring (ABPM) or tele-monitoring, may be advantageous in the near future for further improvement of patient adherence and BP control.

In the treatment of chronic diseases, using drugs with a very good tolerability increase patients' compliance [15]. In our study, only $3 \%$ of the patients discontinued the antihypertensive therapy, and the rate of therapy discontinuation was similar in the HBPM-group as compared to the non-HBPM-group.

This observational study further shows that using a combination therapy composed by two drugs with addictive effect, such as irbesartan and hydrochlorothiazide, lead to a better blood pressure control than a high-dose monotherapy. Evidence from clinical trials indicates that most patients will need more than one agent to achieve their BP goal, and a meta-analysis published in 2009 showed that a combination therapy is far more efficient than doubling the dose of antihypertensive monotherapy [16]. The combination of irbesartan and hydrochlorothiazide has proven to lead to a better BP control than the monotherapy alone, with a good safety profile [17], reduction of microalbuminuria [18], and a better nephroprotection [19].

The present study has several limitations: First, the study is observational and the follow-up was limited to a short-term period. Second, the existence of a bias in the study cannot be ruled out given the selection criteria that were followed to include patients in the HBPM group. Third, the choice of antihypertensive therapy was not determined by an experimental plan but solely is a result of the treatment decision by the physician in charge. Due to lack of randomization in our study the results are hypothesis generating, not confirmatory, and therefore have to be interpreted with caution. In contrast to many randomized controlled trials, our study had no exclusion criteria and the results mirror the then contemporary clinical practice. Even if we acknowledge that ABPM has a better prognostic value than HBPM, we couldn't use ABPM as many GPs did not offer it at the time of the study. Finally, the study was conducted in Switzerland and the results may not be generalizable to other countries.

\section{Conclusion}

In a large Swiss population of patients with arterial hypertension, we observed that clear information and training with focus on blood pressure measurement and direct involvement of patients by using HBPM leads to an improvement in blood pressure control. Treatment with irbesartan alone and in combination with hydrochlorothiazide was well tolerated and markedly reduced blood pressure.

\section{Acknowledgements}

The study was supported by Sanofi-Aventis (Suisse) SA, Vernier, Switzerland. Data collection and data management were conducted by an independent clinical research organization. 


\section{Kidney Blood Pressure Research}

\begin{tabular}{l}
\hline Kidney Blood Press Res 2018;43:979-986 \\
\begin{tabular}{l|l} 
DOI: 10.1159/000490687 & (c) 2018 The Author(s). Published by S. Karger AG, Basel \\
Published online: 19 June, 2018 & www.karger.com/kbr
\end{tabular} \\
\hline
\end{tabular}

Spirk et al.: FIRST: Improving Awareness and Blood Pressure Control

\section{Disclosure Statement}

Dr. Sudano received speaker fee and research support by Sanofi-Aventis (Suisse) SA, Vernier, Switzerland. Dr. Spirk is an employee of Sanofi-Aventis (Suisse) SA, Vernier, Switzerland. No other conflict of interest was reported from the authors regarding the content of this manuscript.

\section{References}

1 Ferrari P, Hess L, Pechere-Bertschi A, Muggli F, Burnier M: Reasons for not intensifying antihypertensive treatment (RIAT): a primary care antihypertensive intervention study. J Hypertens 2004;22:1221-1229.

-2 Berlowitz DR, Ash AS, Hickey EC, Friedman RH, Glickman M, Kader B, Moskowitz MA: Inadequate management of blood pressure in a hypertensive population. N Engl J Med 1998;339:1957-1963.

-3 DiMatteo MR, Giordani PJ, Lepper HS, Croghan TW: Patient adherence and medical treatment outcomes: a meta-analysis. Med Care 2002;40:794-811.

4 Kravitz RL, Melnikow J: Medical adherence research: time for a change in direction? Med Care 2004;42:197199.

-5 Krousel-Wood MA, Muntner P, Islam T, Morisky DE, Webber LS: Barriers to and determinants of medication adherence in hypertension management: perspective of the cohort study of medication adherence among older adults. Med Clin North Am 2009;93:753-769.

-6 Márquez-Contreras E, Martell-Claros N, Gil-Guillén V, de la Figuera-Von Wichmann M, Casado-Martínez JJ, Martin-de Pablos JL, Figueras M, Galera J, Serra A, Compliance Group of the Spanish Society of Hypertension (SEE): Efficacy of a home blood pressure monitoring programme on therapeutic compliance in hypertension: the EAPACUM-HTA study. J Hypertens 2006;24:169-175.

7 van Onzenoort HA, Verberk WJ, Kroon AA, Kessels AG, Nelemans PJ, van der Kuy PH, Neef C, de Leeuw PW: Effect of self-measurement of blood pressure on adherence to treatment in patients with mild-to-moderate hypertension. J Hypertens 2010;28:622-627.

8 Stergiou GS, Bliziotis IA: Home blood pressure monitoring in the diagnosis and treatment of hypertension: a systematic review. Am J Hypertens 2011;24:123-134.

-9 Cifkova R, Erdine S, Fagard R, Farsang C, Heagerty AM, Kiowski W, Kjeldsen S, Lüscher T, Mallion JM, Mancia G, Poulter N, Rahn KH, Rodicio JL, Ruilope LM, van Zwieten P, Waeber B, Williams B, Zanchetti A, ESH/ ESC Hypertension Guidelines Committee: Practice guidelines for primary care physicians: 2003 ESH/ESC hypertension guidelines. J Hypertens 2003;21:1779-1786.

10 Mengden T, Vetter H, Tisler A, Illyes M: Tele-monitoring of home blood pressure. Blood Press Monit 2001;6:185-189.

11 Agarwal R, Bills JE, Hecht TJ, Light RP: Role of home blood pressure monitoring in overcoming therapeutic inertia and improving hypertension control: a systematic review and meta-analysis. Hypertension 2011;57:29-38.

12 Parati G, Stergiou GS, Asmar R, Bilo G, de Leeuw P, Imai Y, Kario K, Lurbe E, Manolis A, Mengden T, O'Brien E, Ohkubo T, Padfield P, Palatini P, Pickering TG, Redon J, Revera M, Ruilope LM, Shennan A, Staessen JA, et al.: European Society of Hypertension practice guidelines for home blood pressure monitoring. J Hum Hypertens 2010;24:779-785.

13 Band R, Morton K, Stuart B, Raftery J, Bradbury K, Yao GL, Zhu S, Little P, Yardley L, McManus RJ: Home and Online Management and Evaluation of Blood Pressure (HOME BP) digital intervention for self-management of uncontrolled, essential hypertension: a protocol for the randomised controlled HOME BP trial. BMJ Open 2016;6:e012684.

14 Joffres M, Falaschetti E, Gillespie C, Robitaille C, Loustalot F, Poulter N, McAlister FA, Johansen H, Baclic O, Campbell N: Hypertension prevalence, awareness, treatment and control in national surveys from England, the USA and Canada, and correlation with stroke and ischaemic heart disease mortality: a cross-sectional study. BMJ Open 2013;3:e003423.

-15 Tedla YG, Bautista LE: Drug Side Effect Symptoms and Adherence to Antihypertensive Medication. Am J 


\section{Kidney Blood Pressure Research}

Spirk et al.: FIRST: Improving Awareness and Blood Pressure Control

Hypertens 2016;29:772-779.

16 Wald DS, Law M, Morris JK, Bestwick JP, Wald NJ: Combination therapy versus monotherapy in reducing blood pressure: meta-analysis on 11,000 participants from 42 trials. Am J Med 2009;122:290-300.

$\$ 17$ Lapuerta P, Franklin S: The risks and benefits of initial irbesartan/hydrochlorothiazide combination therapy in patients with severe hypertension. J Clin Hypertens (Greenwich) 2009;11:277-283.

18 Lehnert H, Bramlage P, Pittrow D, Kirch W: Regression of microalbuminuria in type 2 diabetics after switch to irbesartan treatment: an observational study in 38016 patients in primary care. Clin Drug Investig 2004;24:217-225.

-19 McMullan CJ, Lambers Heerspink HJ, Parving HH, Dwyer JP, Forman JP, de Zeeuw D: Visit-to-visit variability in blood pressure and kidney and cardiovascular outcomes in patients with type 2 diabetes and nephropathy: a post hoc analysis from the RENAAL study and the Irbesartan Diabetic Nephropathy Trial. Am J Kidney Dis 2014;64:714-722. 